\title{
The renal complications of HIV
}

T his issue of Nature Reviews Nephrology focuses on the association between HIV infection and kidney disease. The four Reviews illustrate the clinical significance of renal complications in patients with HIV and highlight the importance of highly active antiretroviral therapy (HAART), dialysis and renal transplantation in these patients. However, given that the prevalence of HIV is greatest in low-income regions, what are the renal consequences for individuals who do not have access to these resources?

The advent of HAART and effective prophylaxis for opportunistic infections has dramatically increased survival of patients with HIV. As a result, however, the prevalence of renal complications has increased and in developed countries, many patients with HIV now die from the complications of end-stage renal disease (ESRD). Indeed, HIV-associated nephropathy (HIVAN) is the most common and most aggressive of the spectrum of renal diseases that can occur with HIV infection; patients with HIVAN can progress to ESRD within weeks. However, survival rates for patients who receive dialysis and HAART are similar to those for patients without HIV. These are encouraging findings for patients with ready access to appropriate care, but are only applicable to a limited number of the millions of people infected with HIV.

According to the WHO, 33 million people worldwide are living with HIV. Although only $10 \%$ of the world's population live in sub-Saharan Africa, $67 \%$ of individuals with HIV inhabit this region. In 2007, 2.7 million new cases of HIV infection occurred; 1.9 million of these were in sub-Saharan Africa. By the end of 2007, only $31 \%$ of patients in need of HAART had access to it. Although very little epidemiological data are available on the prevalence of kidney disease in patients with HIV in sub-Saharan Africa, extrapolation of data from the US suggests that renal complications affect between 770,000 and 2.6 million individuals in this region.

Several studies have suggested that HAART improves renal function and prognosis for patients with HIV. However, access to HAART in developing countries is limited not only by financial and human resource constraints, but also by awareness of HIV status. The WHO estimates that $80 \%$ of individuals with HIV infection in sub-Saharan Africa do not know that they are infected. Many individuals are, therefore, diagnosed with advanced disease. Similarly, most sub-Saharan African patients with HIVAN present late with advanced chronic renal failure. Once diagnosed, the majority of these patients do not have access to renal replacement therapy. HIVAN outcomes correlate with the clinical stage of disease, suggesting that early detection improves survival. The Infectious Diseases Society of America recommends that all individuals should be assessed for kidney disease at the time of HIV diagnosis; however, given the resource limitations in sub-Saharan Africa, achievement of this goal is not currently feasible and the reality is that most people with HIV and ESRD in these regions face a very high risk of mortality because of limited access to early diagnosis, HIV therapy, and dialysis.

Prevention of HIV infection is another approach to slow the spread of HIV and reduce the prevalence of HIVassociated renal complications. HIV vaccine and microbicide trials have, in the past, met with disappointment; however, the results of the world's largest phase III vaccine trial should be released later this year, and several products are under development. Promotion of male circumcision in heavily HIV-affected regions of sub-Saharan Africa is also a major focus of several AIDS organizations.

Interestingly, although treatment and prevention are often considered separate entities, a mathematical model was recently used to demonstrate that annual HIV testing of all individuals followed by immediate antiretroviral therapy after diagnosis could reduce the HIV incidence by about 95\% within a decade (Granich, R. M. et al. Lancet $373,48-57 ; 2009)$. Recent research also suggests that early initiation of HAART is associated with improved survival, and may reduce transmission of disease. These findings underscore the public health benefits associated with the implementation of preventative measures, early diagnosis and early initiation of treatment.

So what are the international goals to address the renal component of the AIDS pandemic? For Western countries, early identification of renal dysfunction by screening at a primary health care level combined with research into the mechanisms of HAART-induced nephropathy may further increase life expectancy. In resource-limited regions such as sub-Saharan Africa, education, along with screening efforts, early diagnosis and initiation of HIV treatment currently offers the best chance for reducing the prevalence of AIDS and the incidence of HIV-associated kidney diseases. However, improved understanding of the extent of renal disease in these regions is arguably the most important step-as with these measures comes the propensity for setting new goals and a sense of what needs to be achieved.

doi:10.1038/nrneph.2009.152



Susan J. Allison is the

Editor of Nature

Reviews Nephrology.

Competing interests The author declares no competing interests. 